\title{
Nefropatía diabética
}

Jara Albarrán A. Nefropatía diabética. An Med Interna (Madrid) 2001; 18: 173-174.

En este número de Anales de Medicina Interna, se publica un interesante artículo de Pérez García y cols. (1) en el que llaman la atención sobre el preocupante incremento de la diabetes como causa de insuficiencia renal terminal (IRT), y se preguntan sobre la eficacia del tratamiento sustitutivo de la función renal.

Recordemos que la nefropatía diabética (ND) constituye una de las complicaciones típicas de la microangiopatía diabética, en cuya patogenia influyen diversos factores (2), siendo los dos más importantes, la hiperglucemia y la hipertensión arterial, que combinados va a producir importantes consecuencias clínicas. De hecho un paciente con buen control diabetológico, con HbA1c menor de 7,5\%, es probable que nunca desarrolle nefropatía. Asimismo, la ND suele ir acompañada de retinopatía diabética en menor o mayor grado.

El hecho de que un porcentaje de diabéticos no desarrollen nefropatía, a pesar de un aparentes mal control de su diabetes, ha hecho pensar en factores genéticos de predisposición o de protección para el desarrollo de la ND. Aunque la controversia se mantenga y sea difícil de aclarar, hay que señalar que los estudios microscópicos de Österby (3) no apoyan dicha hipótesis, y las observaciones clínicas, clásicas, de Pirart (4) en mas de 4.000 diabéticos, así como los estudios más modernos como el DDCT (5) reafirman la contundente correlación entre el defectuosos control de la diabetes y el desarrollo de las complicaciones microvasculares, como es la ND. De influir el factor genético, tendrá poca relevancia (2) y seguramente actuaría a través de su influencia en la hipertensión arterial, como, por ej. el gen del enzima ACE.

En la nefropatía diabética (ND), se distinguen 5 fases (2):

1. Hipertrofia renal-Hiperfiltración glomerular. Presente al diagnóstico. Es reversible con buen control. TA normal

2. Normoalbuminuria: excreción urinaria de albúmina (EUA) menor de $20 \mu \mathrm{g} / \mathrm{min}$. (<30 $<\mathrm{g} / 24 \mathrm{~h}$; <30 $\mathrm{mg} / \mathrm{g}$ de creatinina). Dura 5 años o más. La biopsia renal muestra signos de aumento de la membrana basal. Persiste el aumento (20-50\%) del filtrado glomerular que puede normalizarse con control diabético estricto. TA normal.

3. Nefropatía diabética incipiente con EUA de $20-200 \mu \mathrm{g} / \mathrm{min}$. (30-300 mg/24h; 30-300 mg/g de creatinina). Aparece a los 6-15 años del diagnóstico. Membrana basal glomerular más gruesa. Filtrado glomerular aun aumentado, disminuye al aumentar la EUA. Reversible con control glucémico óptimo más IECA. TA comienza a aumentar.

4. Nefropatía establecida con albuminuria $>200 \mu \mathrm{g} / \mathrm{min}(>300$ $\mathrm{mg} / 24 \mathrm{~h} ;>300 \mathrm{mg} / \mathrm{g}$ de creatinina). Aparece después de 15-25 años de diabetes. TA elevada y otros síntomas de nefropatía. Filtrado glomerular desciende un $10 \%$ al año. El control de la TA frena su pro- gresión. Su avance es mayor si la diabetes no está bien compensada.

5. Insuficiencia Renal Terminal (IRT). Avanza la afectación glomerular y clínica relacionada. Hipertensión arterial. Irreversible a pesar del control dibetológico.

En España, según datos recientes (6), la ND se presenta en el 22$26 \%$ de los pacientes con diabetes mellitus tipo 1 (DM-1), 13-14\% con microalbuminuria y $8-12 \%$ con nefropatía establecida, mientras que la prevalencia en paciente diabéticos tipo 2 (DM-2) es similar para la microalbuminuria (23\%) y del $10-12 \%$ para la nefropatía establecida.

El tiempo de latencia entre el diagnóstico de la diabetes y el inicio de la ND es variable, oscilando entre 8 y 15 años para la DM-1 y algo similar, pero más difícil de evaluar, para la DM-2, dado el desconocimiento de la etapa que precede a su diagnóstico clínico.

Aunque ya hemos comentado líneas atrás la influencia patogénica de la hiperglucemia, hay que recordar que el buen control de la DM-1 retrasa o impide la aparición de la ND. En el estudio DCCT en USA (5), se demostró una reducción del desarrollo de microalbuminuria en un $31 \%$ de los pacientes, y el de la proteinuria en un $54 \%$.

Respecto a la DM-2, el estudio UKPDS (7) señala la dificultad para conseguir un buen control, así como la necesidad frecuente de asociar una terapia múltiple.

En las últimas décadas es sorprendente el aumento de los diabéticos, especialmene tipo 2, que acuden a los servicios de Nefrología ante la aparición y progreso de su afectación glomerular que van a requerir un tratamiento sustitutivo específico con hemodiálisis o diálisis peritoneal. En la explicación de este hecho influyen diversos factores. De una parte el gran aumento de la prevalencia de DM-2 relacionada con la dieta, sedentarismo y obesidad. Por otra parte, la mejor asistencia médica hace que sobrevivan más años y en mejor situación para llegar a desarrollar ND y solicitar el oportuna tratamiento. Además, los avances económicos y técnicos permiten una mayor afluencia y mejor asistencia en los servicios de Nefrología.

En USA los diabéticos que llegaban a IRT constituían el 7\% del total en 1974, pero aumentaron al $25 \%$ en 1980 y al 35\% en 1994 , siendo incluso mayor aun en Minnesota (63\%) (8).

Algo semejante ha ocurrido en Europa y en España. Según se menciona en el trabajo de Pérez García y col (1), el porcentaje de diabéticos en IRT en el área sanitaria 1 de Madrid era 19\% en 1994, pero actualmente llega al 30\%. Por tanto, se puede decir que la diabetes mellitus constituye hoy día la primera causa de entrada en el grupo de pacientes que van a necesitar tratamiento sustitutivo de la función renal. Además, la proporción de diabéticos tipo 2 se va incrementando respecto al total, ya que han pasado del $15 \%$ en el 
período 1987-88, al $81 \%$ en 1997-98. También ha aumentado la edad media de 47 a 63 años. En total presentan los datos de 182 diabéticos, 106 varones y 76 mujeres, de los cuales 45 han recibido un trasplante renal. Hay que resaltar la frecuencia de complicaciones entre los diabéticos que están en tratamiento sustitutivo de la función renal, que son oftalmológicas en un 77\%, vasculopatía periférica en un $35 \%$, accidentes cerebrovasculares en el $21 \%$ y el infarto miocárdico en el 17\%. También señalan la alta mortalidad, 89 casos en 10 años, siendo la causa cardiovascular la más frecuente (29\%), poco mayor que las infecciones (27\%); sólo el $54 \%$ sobreviven a los 5 años, siendo la supervivencia algo mejor con hemodiálisis.

Considerando los datos expuestos, el grupo de nefrología del área 1 de Madrid llega a la conclusión (9) de que se hace necesaria la intervención del nefrólogo, lo más precoz posible, ante el inicio de la insuficiencia renal en cualquier tipo de diabetes. ¿En qué va a consistir dicha intervención? En primer lugar, aparte de vigilar la función renal, controlar la tensión arterial (TA) con un tratamiento eficaz, ya que la TA es el factor más importante en la progresión de la ND. Pero también se vigilarán otros factores, como la posibilidad de infecciones, los niveles lipídicos y la aparición o progreso de complicaciones cardiovasculares. ¿Y el control de la diabetes? Naturalmente corresponde al endocrinólogo (diabetólogo) que conjuntamente con el nefrólogo (9) debería seguir interviniendo en el control del diabético con nefropatía: así ayudaría a vigilar (y tratar) simultáneamente otras posible complicaciones como la retinopatía y neuropatía, además de contribuir al mejor control posible de la diabetes, el cual está demostrado que influye en la progresión de la ND $(10,11)$.

Para muchos médicos, incluidos endocrinólogos, el control diabetológico en esas fases es menos importante que el del nefrólogo. Hay que reconocer que es menos vital que la propia diálisis; además bastante tiene el paciente con acudir con bastante frecuencia a un servicio como para también tener que ir a otro. En ese sentido la colaboración interdisciplinaria y administrativa, para facilitar las citaciones y visitas conjuntas, contribuiría a una mejor atención al paciente, mejorando su control glucémico, con consiguiendo una HbA1c más normal o cerca de la normalidad para disminuir la progresión de la ND, evitando hipoglucemias y colaborando en el control de otros factores de riesgo cardiovascular, como la obesidad, niveles lipídicos y de ácido úrico, además de la TA. En la etapa de nefropatía establecida, el control óptimo de la diabetes ya no podrá

\section{Bibliografía}

1. Pérez Gracia R, Rodríguez Benitez P, Dall'Anesse C, Gómez Campderá F, Valderrabano F. Preocupante incremento de la diabetes como causa de insuficiencia renal terminal. Evaluación del tratamiento sustitutivo. An Med Interna (Madrid) 2001; 18: 175-180.

2. Mogensen CE. Microalbuminuria, blood pressure and diabetic renal disease: origin and development of ideas. Diabetologia 1999; 42: 263-85.

3. Österby R. Lessons from Kidney biopsies. Diab Metab Rev 1996; 12: 151-74.

4. Pirart J. Diabetes Mellitus and its degenerative complications: a prospective study of 4,400 patients observed between 1974 and 1973. Diabetes Care 1978; 1: 168-8.

5. The Diabetes Control and Complications Trial Research Group. The effect of intensive treatment of diabetes on the development and progression of long-term complications in insulin-dependent diabetes mellitus. N Engl J Med 1993; 329: 977-86.

6. Esmatjes A. La nefropatía diabética. Dimensión del problema en España. Av Diabetol 1999: 15 (S): 10-2.

7. Turner RC, Cull CA, Frighi V, Holman RR. Glycemic control with diet, sulfonylurea, metformin, or insulin in patients with type 2 diabetes mellitus: progressive requirement for multiple therapies (UKPDS 49). UK Prospective Diabetes Study (UKPDS) Group. JAMA 1999; 281: 2005-12.

8. Luño J, Valderrabano $\mathrm{F}$, ¿Se puede evitar la progresión a la insuficien- hacer retroceder la ND pero sí va a influir en su progresión y en la evolución de la micro y macroangiopatía de los años siguientes, especialmente interesante para disminuir su gran morbimortalidad en la etapa de tratamiento sustitutivo de la función renal, incluso, más aun, si llega al trasplante renal, para evitar entre otras cosas la reaparición de la ND en el riñón trasplantado.

Pero también hay que preguntarse qué ocurriría si esa compensación óptima se consiguiera en la fase de microalbuminuria. Existen numerosos estudios en los que el control diabetológico riguroso unido a la administración de IECA, es capaz de revertir ese estadío de la ND. La combinación de euglucemia y normotensión es la mejor renoprotección $(7,11)$. Incluso se está estudiando si el empleo de IECA antes de esa fase podría ser beneficioso a la larga. Sin embargo, es un hecho, que no en todos los diabéticos se puede conseguir un control óptimo y que, aun consiguiéndolo, el empleo de los IECA u otros hipotensores, no siempre logra impedir el progreso de la ND (12).

Por tanto, las reflexiones actuales acerca de la ND nos llevan a esa preocupación por el incremento de la diabetes como causa de IRT, tal como señalan Pérez García y cols. (1), y a apoyar su propuesta de "desarrollar nuevas estrategias de prevención de la angiopatía y nefropatía en los pacientes diabéticos". Pero además, considerando la falta de reversibilidad de la nefropatía establecida, habría que dirigir la atención hacia la fase inicial de microalbuminuria o aun antes, y proponer a la sociedad una implementación eficaz de medidas de control de la diabetes mellitus para que los diabéticos puedan disponer de una opción real para evitar la aparición de la ND y demás complicaciones diabéticas. De ahí la necesidad de ayuda para la investigación (clínica y básica) en torno a nuevas técnicas de tratamiento "curativo" como pudiera ser el páncreas artificial miniaturizado (ya tenemos los sensores al alcance de la mano (13), los trasplantes de islotes (el año 2000 puede haber marcado un hito en este sentido (14) o los implantes de células genéticamente modificadas para la producción de insulina (15), quizá no tan cerca del éxito como continuamente señalan los medios.

A. JARA ALBARRÁN

Servicio de Endocrinología Hospital General Universitario Gregorio Marañón. Madrid

cia renal terminal en la nefropatia diabetica? An Med Interna (Madrid) 1998; 15: 567-71.

9. Gómez Campderá FJ, Luño J, García de Vinuesa S, Valderrabano F. ¿Por qué es necesaria la remisión precoz a la consulta del nefrólogo de los pacientes con nefropatía diabética ? Rev Clin Esp 1999; 199: 82-3.

10. Alaveras EAG, Thomas SM, Sagriotis A, Viberti GC. Promotors of progression of diabetic nephropathy: the relative roles of blood glucose and blood pressure control. Nephrol Dial Transplant 1997; 12: 71-4.

11. Cooper M E. Pathogenesis, prevention, and treatment of diabetic nephropathy. Lancet 1998; 352: 213-19.

12. Mathiesen ER, Hommel E, Hansen HP, Smidt UM, Parving HH. Randomised controlled trial of long term efficacy of captopril on preservation of kidney function in normotensive patients with insulin dependent diabetes and microalbuminuria. Brit Med J 1999; 319: 24-5.

13. Pickup J. Sensitive glucose sensing in diabetes. Lancet 2000; 355: 426-27.

14. Shapiro AMJ, Lakey JRT, Ryan EA, Korbutt GS, Toth E, Warnock GL, et al. Islet Transplantation in seven patients with type 1 Diabetes Mellitus using a glucocorticoid-free imnunosuppressive regimen. $\mathrm{N}$ Engl J Med 2000; 343: 230-8.

15. Soria B, Roche E, Berná G, León-Quinto T, Reig JA, Martin F. Insulinsecreting cells derived from embryonic stem cells normalize glycemia in streptozotocin-induced diabetic mice. Diabetes 2000; 49: 157-62. 